\title{
Am Ende der kritischen Soziologie
}

\author{
Niklas Luhmann \\ Fakultät für Soziologie, Universität Bielefeld, Postfach 86 40, D-4800 Bielefeld
}

Zusammenfassung: Die kritische Soziologie der bürgerlichen Gesellschaft wird heute nicht durch politische, sondern durch wissenschaftliche Innovationen verunsichert: Provozierender als das Ende des Sozialismus stellt das Ende der ontologischen Weltkonzeption sie in Frage. Der Beitrag schlägt den Übergang zu einer genau darauf eingestellten Theorie der Beobachtung zweiter Ordnung vor. Dabei können Anregungen zu Latenzaufklärung und Ideologiekritik aufgenommen und reorganisiert werden. Die epistemologischen Weiterungen betreffen jedoch den Realitätsbegriff selbst und reichen damit hinaus über das, was innerhalb der kritischen Soziologie bisher formuliert worden ist. Die Differenzen lassen sich unter anderem an der klassischen Krisensemantik und an den Paradoxien eines dazu passenden Anspruchs „kritischer“, nämlich jeweils eigener Überlegenheit demonstrieren.

Die im folgenden präsentierten Überlegungen gehen nicht davon aus, daß die Soziologie durch die Realereignisse beim Zusammenbruch sozialistischer Politik- und Wirtschaftssysteme zu einer Änderung ihrer Perspektiven gezwungen werden könnte. So direkt kann die Soziologie in ihren konstitutiven Einstellungen nicht betroffen werden. So unmittelbar kann sie nicht auf unbestreitbare Tatsachen reagieren. Aber es mag tieferliegende Gründe geben, aus denen die bürgerliche Soziologie das Spiel von Kritik und Krise nicht mehr weiterspielen kann. Es gibt eine mehr als zweihundertjährige Tradition dieses Stils und man kann sie als ,bürgerlich“ bezeichnen, obwohl die Soziologie selbst es bevorzugt, sich als Opposition darzustellen und gegen diejenigen $z u$ Felde zu ziehen, die von ihr als Vertreter der bürgerlichen Gesellschaft charakterisiert werden. Die Semantik von Kritik und Krise war aber ein Phänomen der bürgerlichen Gesellschaft selbst, wenn man eine Gesellschaftsepoche so bezeichnen darf, die sich mit dem Zusammenbruch der Adelswelt konfron* tiert sah, aber nicht sicher ausmachen konnte, welche Strukturen an deren Stelle getreten waren oder treten würden. ${ }^{1}$ Krisendiagnose und kritische Einstellung $\mathrm{zu}$ den sich anscheinend durchsetzenden Strukturen bestimmen seitdem progressive und konservative Stellungnahmen. Der Zeitgeist scheint durch „Entzweiung“, durch „Ultrageist", durch Absolutsetzung von Parteistandpunkten bestimmt zu sein. ${ }^{2}$ Die Zukunft läßt sich unter diesen

\footnotetext{
${ }^{1}$ Hier genügt der Hinweis auf Koselleck (1959).

${ }^{2}$ So lamentierend und dann auf Religion setzend Friedrich Schlegel (1823: 599f) zum Zusammenhang von: Mangel an Beurteilungskraft, Neigung zur Kritik und Krisenhaftigkeit.
}

Umständen nur unsicher und nicht einhellig beurteilen, und sicher ist nur, daß sie nicht so sein wird wie die Vergangenheit.

Die Soziologie setzt diese Tradition, die sie vorfindet, fort. Was sie neu versucht, ist: sich als Wissenschaft zu etablieren und die Gesellschaft als ihr Objekt zu fixieren. Das zwingt sie, logisch und methodisch, zur Externalisierung ihres eigenen Standpunktes. Erst in jüngster Zeit findet man eine methodologische Kritik dieses Ausgangspunktes, eine Kritik der Prämisse eines beobachtungsunabhängig gegebenen Objekts Gesellschaft. Das betrifft schon die Voraussetzungen empirischer Forschung, erst recht aber - und nur das ist unser Thema - die soziologische Gesellschaftskritik. Man mahnt bereits an: Kritiker müßten über ihren eigenen gesellschaftlichen Standort in ihrer eigenen Theorie Auskunft geben. ${ }^{3}$ Aber wie wäre das möglich? Und was würde es nützen?

Ein erster auffälliger Sachverhalt ist: daß in der vorsoziologischen und in der soziologischen Literatur Kritik mit einer Krisendiagnose begründet wird. Wie verschwommen auch immer der Begriff der Krise gehandhabt werden mag, seitdem er seinen medizinischen Anwendungsbereich überschritten hat: in jedem Falle bezeichnet er eine vorübergehende Lage, also einen bedenklichen Zustand, der nicht im „Wesen“, in der "Natur“, in der „Struktur" des Systems begründet ist. ${ }^{4}$ Wer von Symptomen einer Krise spricht, hat die Hoffnung noch nicht aufgegeben. Gerade in der Spannung zwischen Gefahr und Hoffnung liegt der Ap-

${ }^{3}$ So Thomas C. Heller (1984: 170f). Im übrigen ist dieses Desiderat nicht gerade neu, aber offenbar schwer zu erfüllen. Siehe z. B. Linguet (1764).

${ }^{4}$ Hierzu begriffsgeschichtlich Reinhart Koselleck (1982). 
pellcharakter des Begriffs. Die krisenhaften Erscheinungen der Gegenwart werden auf Fehlentwicklungen, vor allem des Industriekapitalismus, zurückgeführt, die man korrigieren kann. Noch in den 70er Jahren konnte man lesen, daß die ökologischen Probleme der modernen Gesellschaft ein Phänomen des profitsüchtigen Kapitalismus seien und unter sozialistischen Bedingungen nicht auftreten würden. Es muß gleichsam eine gute Gesellschaft hinter der Gesellschaft geben, auf die man Strukturen und Effekte zurückdirigieren kann.

Die Rhetorik der Krise erfordert mithin einen Gesellschaftsbegriff, der auf zwei Ebenen angesiedelt ist und so die Entzerrung einer Paradoxie ermöglicht. An einem an sich lebensfähigen System ist etwas schlimm geraten; oder ins Normative gewendet: die für die Fortsetzung geordneter Gesellschaft notwendigen Normen werden mißachtet. Mit dieser Voraussetzung zweier Ebenen eignet sich der Krisenbegriff zur Selbstbeschreibung einer Gesellschaft, die sich in einer Übergangsphase befindet und den Zusammenhang von Strukturen und Folgen noch nicht voll begreifen kann. Auch wenn heute davon gesprochen wird, $\mathrm{da} ß$ die moderne wissenschaftlich-technische Zivilisation ihr eigenes Ende herbeiführen könne, ist dieser Krisenbegriff vorausgesetzt. Es kann sein, es muß nicht sein. Die Gesellschaft selbst ist ein System hinter den Problemen, die zur Krise geführt haben, aber anders gelöst werden könnten. Noch heute ermutigen deshalb Krisendiagnosen zu sozialen Bewegungen, die gegen unakzeptable gesellschaftliche Verhältnisse protestieren. ${ }^{5}$

Zugleich stimulieren aber Bemühungen um eine Erklärung der Krise Gesellschaftsbeschreibungen, die die Phänomene auf Strukturen zurückführen und das Wissen um die Realitäten der modernen Gesellschaft komplexer und differenzierter werden lassen. Wie in einem unbeabsichtigten perversen Effekt kommt bei ständigen Krisendiagnosen nach und nach heraus, daß es sich gar nicht um Krisen handelt, sondern um die Gesellschaft selbst. Das gilt besonders in dem Maße, als die ökologische Problematik die alte Verteilungsproblematik verdrängt und damit die Aufmerksamkeit von den bürgerlichen Ideen der Freiheit und Gleichheit auf das Betroffensein der menschlichen Körper umgelenkt wird. Nicht Emanzipation aus der Unmündigkeit (welche Zumutung der Philosophen an andere, sich so beschreiben zu lassen!) ist das Pro-

${ }^{5}$ Siehe, dies beschreibend begleitend, unter anderen Wilfried von Bredow/Rudolf H. Brocke (1987). blem, sondern Leben und Überleben. Auch dieser Schritt wird noch unter dem Konzept einer „Krise“ vollzogen. Aber in dem Maße, als diese Krise soziologisch erklärt wird, entsteht eine Gesellschaftstheorie, die die Krisenphänomene nicht mehr nur als vorübergehend behandeln, nicht mehr nur auf falsches Bewußtsein oder falsche Politik zurückführen kann, sondern sie als strukturelle Effekte der modernen Gesellschaft begreifen muß. Und wenn eine solche realistische Begegnung der Gesellschaft mit sich selbst erreicht ist: was könnte dann noch Kritik besagen?

Gleich welcher Herkunft und welcher theoretischen Ausstattung, der "kritische Rationalismus", die „kritische Theorie“ usw. hatten immer Attitüden des Besserwissens angenommen. Sie stellten sich als konkurrierende Beschreiber mit tadelfreien moralischen Impulsen und mit besserem Durchblick vor. Wie immer vorsichtig formuliert und wie immer den Anforderungen an Wissenschaftlichkeit genügend, ihre Perspektive war die eines Weltbeobachters erster Ordnung. Man bot eine konkurrierende Gesellschaftsbeschreibung an und stand damit vor der Aufgabe, zu erklären, wieso andere in derselben Welt diese Auffassungen nicht (oder noch nicht) teilen. Man konnte dann sagen, sie hätten Popper nicht sorgfältig genug gelesen und seien wissenschaftstheoretisch nicht auf der Höhe der Zeit; oder der Verblendungszusammenhang der eigenen Interessen verhindere die Einsicht in die gesellschaftlichen Verhältnisse. Dabei wurde es jedoch immer schwieriger, die eigenen Ausgangspunkte kritikfrei zu setzen. Schließlich hatten schon David Hume, Adam Smith und andere gelehrt, $\mathrm{da} \beta$ die Vernunft verlange, da $\beta$ man beobachte, wie andere einen beobachten. Wenn das nicht beachtet wird, ist unter modernen Bedingungen (aber wie werden die reflektiert?) nur noch Sektenbildung möglich. Sowohl der „,kritische Rationalismus" als auch die "kritische Theorie" haben diesem Schicksal nicht entrinnen können - was immer man über die Zahl ihrer Anhänger dann noch ausmachen kann. Sie haben statt auf besseres Wissen aufs bessere Verfahren gesetzt, sie haben, wie man heute sagt, ihre Position ,prozeduralisiert". Das hat die Anstößigkeit gemildert, hat aber im Prinzip nichts geändert. Und im Prinzip heißt: daß es sich um eine Perspektive des Weltbeobachters erster Ordnung handelt.

Immerhin waren andere Denkmöglichkeiten inszeniert und unübersehbar präsent. Man konnte versuchen, sie aus der Wissenschaft auszuschließen und zur Literatur zu erklären. Aber das Zitiernetz 
der Wissenschaft selbst führt eine solche Strategie ad absurdum. Eine andere Strategie, wie sie mit Nachdruck und Eleganz von Jürgen Habermas vertreten wird, arbeitet mit gesellschaftspolitischen Positionszuweisungen. Mehr und mehr kompensiert dann aber das Beschreiben derjenigen, die angeblich gesellschaftskonform, affirmativ, konservativ usw. denken, das Stagnieren der eigenen Theorieentwicklung. Auf gut marxistischen Bahnen wird Ideologiekritik zum Schwerpunkt des eigenen Denkens, und die eigene Gesellschaftsbeschreibung verlagert sich in immer raffiniertere Bemühungen, dem fremden Theoriegut gerecht zu werden und zugleich zu erklären, auf Grund welcher gesellschaftlichen Bedingungen andere nicht in der Lage sind, die Gesellschaft (inclusive sich selber) so zu sehen, wie es von den Kritikern für richtig gehalten wird. In dieser Hinsicht kann freilich die Theorieleistung von Marx kaum überboten werden. Es verändert sich nur der gesellschaftliche Kontext des Angebots. In dem Maße, als „,konservative" Einstellungen (das heißt: Einstellungen gegen die Ideen der Französischen Revolution) an Überzeugungskraft verlieren und in dem Maße, als die Vorstellungswelt des (politischen) Liberalismus durch Übertragung auf wirtschaftliche Sachverhalte an dynamischer Stabilität gewinnt, nimmt die Faszination der Kritiker durch ihre Gegner zu. Sie mußten schließlich das Etikett „neokonservativ" erfinden (das heißt: die Linie von traditionsgebunden zu konservativ ins Paradoxe verlängern), um ihre Gegner zu formieren und sich selbst weiterhin im Geschäft der Kritik zu halten. Aber kein Denker von Rang würde sich selbst als „neokonservativ" beschreiben. ${ }^{6}$ Es handelt sich offensichtlich um eine Fremdbeschreibung, die sich gar nicht die Mühe macht, zu beobachten, wie der Beschriebene sich selber beobachtet. Dann aber ist die Dauerproduktion von Dissens im Zeichen vernünftiger Verständigung die konsequente Endposition dieser großen bürgerlichen Tradition von Krise und Kritik.

Am Ende dieser Tradition zeigt sich an ihr selbst bereits die Form, in der sie, wie man seit Hegel sagt, ,aufgehoben“ werden kann. Wenn es ohne-

6 Jedenfalls enthält der Artikel „Neokonservativismus“ von Helmut Dubiel in Pipers Handbuch der politischen Ideen (1987), dafür keinen Hinweis, und er stammt auch nicht (man mag das bedauern) von einem „Neokonservativen". Vielleicht handelt es sich also bei "Neokonservativen" um Wesen wie die westafrikanischen Leopardenmenschen, von denen viel geredet wird, die es aber möglicherweise gar nicht gibt. hin nur noch darum geht, Beobachtungen zu beobachten und Beschreibungen zu beschreiben, könnte dann nicht die gesamte Theoriekonstruktion auf eine Ebene der Beobachtung zweiter Ordnung überführt werden? Die Realitätsgarantie läge dann nur noch im faktischen Stattfinden des Beobachtens. Das Beobachten zweiter Ordnung ist immer auch ein Beobachten erster Ordnung, aber es konzentriert sich dabei auf ein Beobachten von Beobachtern, was als Selbstbeobachtung oder als Fremdbeobachtung durchgeführt werden kann. Entsprechend ist die Welt nicht mehr eine Dinggesamtheit (universitas rerum), sondern ein Korrelat des Beobachtens von Beobachtungen.

Sowohl die Schleifen-Kybernetik als auch die Systemtheorie bieten hierfür ausgearbeitete Konzepte an. ${ }^{7}$ Auch die Semiotik bewegt sich in diese Richtung, wenn sie sich nicht mehr damit begnügt, den Zeichen die Referenz zu bestreiten, sondern das Zeichen als Unterscheidung von Bezeichnendem (signifiant) und Bezeichnetem (signifié) selbst zu bezeichnen versucht. ${ }^{8}$ Dabei sind dann aber schon Theorieentwürfe vorausgesetzt, die man den Erben ,kritischer“ Theorien vielleicht nicht zumuten kann - etwa der kybernetische Kontrollbegriff oder die System/Umwelt-Unterscheidung der Systemtheorie oder der ins „konstruktivistische“ tendierende Zeichenbegriff der Semiotik. Man könnte das Terrain in all diesen Hinsichten jedoch offen halten und zunächst nur den Zugewinn klären, der mit dem Übergang auf eine Ebene der Beobachtung zweiter Ordnung und, daraus folgend, mit logisch strukturreicheren Theorieangeboten verbunden ist. ${ }^{9}$

Der Beobachter zweiter Ordnung kann sich, wie gewohnt, zum Beobachter erster Ordnung „kritisch" einstellen, er kann sich ihm gegenüber ab-

7 Siehe z. B. Gotthard Günther (1976: 329-351); Humberto R. Maturana (1982: 64); Heinz von Foerster (1981), (1985); Ranulph Glanville (1988); Niklas Luhmann et al. (1990). Wissenschaftssoziologisch wäre die vor allem durch Heinz von Foerster organisierte enge persönliche Bekanntschaft und Kooperation der hier mit Namen genannten Forscher erwähnenswert, die an einer Epistemologisierung der Naturwissenschaften und damit auch an einer Naturalisierung der Epistemologie interessiert sind.

${ }^{8}$ Siehe das Konzept einer selbstkritischen second semiotic bei Dean MacCannell/Juliet Flower MacCannell (1982).

${ }^{9}$ „Logisch strukturreicher" soll einfach heißen, daß die Verwendungsbeschränkungen des logischen Operators der Negation erweitert werden. 
lehnend oder belehrend verhalten, kann Übernahmeangebote unterbreiten (was auch wir soeben tun) - aber er muß sich in seinem eigenen Beobachten beobachten bzw. beobachten lassen. Er muß seine Instrumente offenlegen, muß sich WieFragen stellen.

Ebenso kann man das Interesse an „latenten“ Strukturen und Funktionen weiterverfolgen. Man kann weiterhin behaupten, daß das Bewußtsein seine Funktionsbedingung in etwas „Unbewußtem" hat, so wie das Auge einen blinden Fleck oder wie die Logik eine Paradoxie benötigt, um funktionieren zu können. Aber auch diese Einsicht ist, wie Linguisten sagen, ,,autologisch“ zu verwenden. Sie trifft auch auf den zu, der sie an anderen praktiziert. Nur wenn das akzeptiert wird, kann man dem Vorwurf entgehen, Beobachtung hinter dem Rücken sei schlechtweg Betrug. ${ }^{10}$

Auch Verfolger aller Arten, Moralisten, soziale Bewegungen, religiöse Eiferer kann man auf diese Weise mit Gewinn betrachten. Was sie als Eigenschaften ihrer Opfer sehen, sind sie selber, sind ihre eigenen Beobachtungen. René Girard hat entsprechende Annahmen zu einer Theorie des Imitationskonfliktes ausgearbeitet. ${ }^{11}$ Allerdings darf das nicht zu dem Fehler führen, nunmehr die Verfolger zu Opfern zu machen und sie zu verfolgen; oder die Moralisten nun ihrerseits moralisch zu verurteilen. Der theoretische Vorrang der Operation des Beobachtens führt vielmehr nur zu der Frage, welche Folgen es hat, wenn so beobachtet wird, wie beobachtet wird - und nicht weiter.

Schließlich läßt sich auch die gesamte erkenntnistheoretische Tradition auf der Basis einer ontologischen Metaphysik einbauen. Sie besagt auf der Ebene der Beobachtung zweiter Ordnung, da $B$ ein Beobachter versucht, sich mit anderen Beobachtern über bestimmte zweiwertige Schemata, nämlich Sein/Nichtsein und wahr/unwahr zu verständigen, um dann festzustellen, ob deren Beobachtungen mit seinen übereinstimmen oder nicht, und wer, wenn nicht, im Irrtum ist, also lernen muß.

${ }^{10}$ So Michel Serres (1985: $41 \mathrm{ff}$ ), wo das Beobachten von Beobachtern als Betrug an Betrügern dargestellt wird. Aber das heißt ja nur, daß man auf diese Weise nie wieder zur Unmittelbarkeit des Weltglaubens der eigenen Sinne zurückfindet.

${ }^{11}$ Siehe vor allem Girard (1982). Im übrigen kann man an der Entwicklung dieser Theorie gut beobachten, wie ein ursprünglich fast psychoanalytisches Argument in eine Theorie der sich selbst organisierenden Zirkel übergeht.
Nur ist auf der Ebene der Beobachtung zweiter Ordnung klar, daß auf diese Weise, weil ja die Wahl der Schemata vorangeht, nicht festgestellt werden kann, wie die Welt wirklich beschaffen ist, sondern nur, wie sie intersubjektiv übereinstimmend konstruiert werden kann mit der Folge, daß die Kommunikation fortgesetzt werden kann und nicht mangels Verständigungsmöglichkeiten abgebrochen werden muß. ${ }^{12}$

Der damit gewonnene Strukturreichtum möglicher Theorien hängt $a b$ von einer radikal operativen Basis, die auch die Theorieentwicklung selbst in ihren Bann zieht, also ,autologisch" funktioniert. Das Beobachten selbst hat höheren Realitätswert als der Beobachter. Der Beobachter (das Individuum zum Beispiel oder ein System sozialer Kommunikation) ist nur ein Konstrukt zur Ermöglichung weiterer anschlußfähiger Beobachtungen. Das spezifisch moderne Individuum ist weder „ein Mensch“ des 18. Jahrhunderts und schon gar nicht „das Menschenbild", an dem, wie die Kritiker verlangen, die Gesellschaft ausgerichtet werden solle. ${ }^{13}$ Die Individualität im spezifisch modernen Sinne verlangt vielmehr dauernde Selbstbeobachtung im strengen Sinne, das heißt die ständige Selbstkonfrontation mit der Frage, wie bin ich (nicht bloß: wer bin ich!), also mit der Beobachtung des Beobachters, der man selber ist, im Hinblick auf die Eigenart und Instrumentierung seines Beobachtens, die auch anders gewählt werden könnten. ${ }^{14}$ Erst durch Beobachten des Beobachtens distanziert sich ein "Subjekt" von der Welt und gewinnt eine Reflexionskapazität, die nur als

12 Auch das Konzept, daß auf die damit gegebene Unsicherheit schon reagiert, sei wenigstens anmerkungsweise noch erwähnt. Auf der Linie Bacon-HobbesLocke-Vico wird argumentiert: Auch wenn man nicht weiß, in welcher Welt man gemeinsam mit anderen lebt und ihrer Lehrautorität nicht mehr traut, kann man immer noch den anderen beim Herstellen, Machen, Produzieren, Experimentieren beobachten, also als Beobachter beobachten. Wenn man dann sein Herstellen copieren und wiederholen kann und zum selben Resultat kommt, führt das zu intersubjektiv gewissem Wissen. Allerdings weiß man heute auch, da $B$ die Wissenschaft sich praktisch kaum mit Replikationen befaßt. Es genügt ihr, sich auf die Theorie des Beobachtens von Beobachtungen zu verlassen.

${ }^{13}$ Mit der Folge, daß man fragen muß: durch wen?, und über diese Frage in ein unbehebbares politisches Mißtrauen geführt wird.

${ }^{14}$ Manche nennen das heute mit hilfreicher Verständnislosigkeit „Poststrukturalismus". 
Kapazität in der Welt, nicht als Kapazität der Welt konstituiert werden kann, weil sie nur mit Hilfe einer Unterscheidung praktiziert werden kann. ${ }^{15}$ Ebenso gliedert sich hier das schon lange diskutierte Problem des Fremdverstehens ein. Man beobachtet den anderen als Beobachter (und wieder: nicht bloß als einen anderen Menschen, als ein anderes Lebewesen gleicher Art); und das heißt: über Kommunikation ist zu entscheiden im Hinblick darauf, wie andere beobachten und ob man deren Beobachtungsweise bestätigen oder durchkreuzen oder therapieren will. Das war im übrigen die Botschaft von Erving Goffman, der wohl deshalb weder von den empirischen noch von den kritischen Soziologen als einer der ihren akzeptiert werden konnte.

(Es lohnt an dieser Stelle eine kleine Anmerkung über ein unbekanntes, weil nie publiziertes Stück Soziologiegeschichte. Helmut Schelsky hatte sich die Frage gestellt, wie eine Dauerreflexion auszuhalten sei, und seine Vermutung war: nur durch Institutionalisierung. ${ }^{16}$ Gotthard Günther hatte sich die Frage gestellt, wie Du-Subjektivität (also: der andere als Beobachter) beobachtet und beschrieben werden könne, und seine Vermutung war: nur mit Hilfe einer mehrwertigen Logik. ${ }^{17}$ Schelsky Begriff der Institution war jedoch viel zu konkret, weil anthropologisch-bedürfnisorientiert formuliert; und Günthers Überlegungen zu Strukturproblemen einer mehrwertigen Logik führten zu extrem abstrakten, inhaltsleeren Formalismen. So kam es trotz intensiver Diskussion nicht $\mathrm{zu}$ formulierbaren Ergebnissen. Für eine ausgearbeitete Theorie war die Zeit gegen Ende der 60er Jahre noch nicht reif. Das Terrain blieb vorerst

${ }^{15}$ Siehe Günther Beiträge (1976) Bd. 1, S. 339 i. V.m. 319. Günther nennt dies Beobachten des Beobachtens „founding relation“. Siehe auch George Spencer Brown (1979: 105). Im übrigen finden wir uns hier in der Welt des späteren Wittgenstein.

${ }^{16}$ Siehe Helmut Schelsky (1957: 153-174).

${ }^{17}$ Siehe Gotthard Günther (1976-1980). Mit Hinweisen zu „Du-Subjektivität“ im Index.

18 „So wird es deutlich, daB Subjekt und Objekt precaire/ scheinbare/Akzidenzen sind“, liest man in Novalis" Fichte-Studien, zit. nach: Novalis (1978: 24) Bd. 2.

${ }^{19}$ Von Ironie als Überbrückung persönlich erfahrener Diskrepanzen, Distanz zu den Weltgeschäften, denen man doch sehr nahe steht, spricht Friedrich Schlegel und warnt davor, dies für Indifferenz zu halten. Siehe Schlegel (1823: 623). den kritischen, individuell leichter aneignungsfähigen Ambitionen der Soziologie überlassen.)

Was wären die Konsequenzen eines solchen Übergangs von kritischer Soziologie zur Beobachtung zweiter Ordnung? Eine Antwort auf diese Frage ist nicht sicher auszumachen, solange man es nicht mit hinreichender Konsequenz versucht. Es wird nicht einfach um einen „Paradigmawechsel“ gehen, denn dieser müßte auf der Ebene von Theorien vollzogen werden. $\mathrm{Zu}$ vermuten ist, daß die Form der Distanz zwischen Soziologie und Gesellschaft sich ändert. Die Form der Distanz! Es geht also nicht einfach um Zunahme der Distanz. Vielmehr wird die Unterscheidung von Subjekt und Objekt prinzipiell aufgegeben, die es dem Subjekt ermöglicht hatte, sein Objekt zu beurteilen und zu verurteilen, ohne sich selbst zu treffen. Die Soziologie ist in der Gesellschaft für Beobachtungsfunktionen freigestellt, und gerade ihre Autonomie ist die Form, mit der sie in das rekursive Netzwerk des Beobachtens von Beobachtungen eingespannt ist, das heißt: gesellschaftlich existiert.

Ein erster Versuch dieser Art war die romantische Ironie gewesen - mit Verzicht auf die Unterscheidung Subjekt/Objekt ${ }^{18}$, mit hoher Betroffenheit durch die Verhältnisse (insbesondere nach der Französischen Revolution) ${ }^{19}$ und mit dem Versuch, doch an einem immer ferner rückenden Einheitsgedanken festzuhalten. Aber Ironie ist nur ein Stilmittel, eine Duftmarke, die man im Terrain der Kritiker absetzt (Man hat sie beobachtet!), aber kein technisches Mittel der Theoriekonstruktion.

Hier wäre eher an eine Unwahrscheinlichkeitshypothese nach dem Muster von Evolution und Negentropie zu denken, also an ein theoriemotivierendes Staunen darüber, daß überhaupt etwas zustandekommt.

\section{Literatur}

Bredow von, W./R. H. Brocke, 1987: Krise und Protest: Ursprünge und Elemente der Friedensbewegung in Westeuropa, Opladen.

Dubiel, H., 1987: Neokonservativismus. S. 475-479 in: Pipers Handbuch der politischen Ideen Bd. 5, München.

Foerster, H. von, 1981: Observing Systems, Seaside Ca1.

Foerster, H. von, 1985: Sicht und Einsicht: Versuche zu einer operativen Erkenntnistheorie, Braunschweig.

Girard, R., 1982: Le Bouc émissaire, Paris.

Glanville, R., 1988: Objekte, Berlin.

Günther, G., 1976: Formal Logic, Totality and The Super-additive Principle. S. 329-351 in: ders., Beiträge zur Grundlegung einer operationsfähigen Dialektik Bd. 1, Hamburg. 
Günther, G., 1976-1980: Beiträge zur Grundlegung einer operationsfähigen Dialektik, 3 Bde., Hamburg.

Heller, T. C., 1984: Structuralism and Critique, Stanford Law Review 36: 127-198.

Koselleck, R., 1959: Kritik und Krise: Die Pathogenese der bürgerlichen Welt, 2 . Aufl. Freiburg.

Koselleck, R., 1982: Geschichtliche Grundbegriffe: Historisches Lexikon zur politisch-sozialen Sprache in Deutschland Bd. 3, S. 617-650.

Linguet, S., 1764: Le Fanatisme des Philosophes, London-Abbeville.

Luhmann, N. et al., 1990: Beobachter: Konvergenz der Erkenntnistheorien?, München.

MacCannell, D./J. Flower MacCannell, 1982: The Time of the Sign: A Semiotic Interpretation of Modern Culture, Bloomington Ind.
Maturana, H. R., 1982: Erkennen: Die Organisation und Verkörperung von Wirklichkeit: Ausgewählte Arbeiten zur biologischen Epistemologie, Braunschweig.

Novalis, 1978: Fichte-Studien, in: H.-J. Mähl/R. Samuel (Hrsg.), Werke, Tagebücher und Briefe Friedrich von Hardenbergs, Darmstadt, Bd. 2.

Schelsky, H., 1957: Ist die Dauerreflexion institutionalisierbar?, Zeitschrift für evangelische Ethik 1: $153-174$.

Schlegel, F., 1984: Signatur des Zeitalters (1823) S. 593-728 in: W. Rasch (Hrsg.), Dichtungen und Aufsätze, München.

Serres, M., 1985: Les cinq sens, Paris.

Spencer Brown, G., 1979: Laws of Form, (Neudruck) New York. 